\title{
An Interactive Pill Box using IoT
}

\author{
Harshitha V, Sandeep K, Swasthika Jain T J
}

\begin{abstract}
As the population on the earth is growing, the long-ranging planning of health and medical facilities are affected. Especially with old-aged people, health issues are more compared with other aged people. The medication given by the doctors to old age people to those health issues is not rememberable. People need to take the pills with a specified dose at a recommended time and frequency especially in case of diabetes and high blood pressure. To overcome the problem an IoT device is designed to remember about their medication time to the old people and their caretakers. The IR sensor present in the system will be continuously monitoring whether medicines has been taken properly by the patient or not. By using the GSM, the caretakers have been notified to their smart phones and watches. So, we design a pillbox which acts as a safety net for patients. The main objective of the system is to inform the patients to take their medicines in time that is prescribed by the doctor and to inform their family members which reduces their work.
\end{abstract}

Keywords: Medication, Disease control, Safety, Pills, IoT, Arduino Nano, Smart Watch and Smart Phone.

\section{INTRODUCTION}

Medicines are a greedy need for many people nowadays. It may be due to the hereditary diseases, or disease attained from different people or blood-borne disease. Diseases do not have constraints of age it may occur in old or young age people. The rate of disease is getting higher and the consumption of antibiotics is escalating. Comparatively, the technology is impressing with agile gadgets. So, in this paper introduction of a smart pillbox which can help people in hospital or in-home who are affected with different diseases, by intimating the family members, medical shop or it may give an alarm at a particular time which has been laid into the gadget. So, the patient can get their dosage regularly in a proper manner and time.

\section{LITERATURE SURVEY}

Some People will obliterate to have proper medicine correctly on time. To help these community a smart med box which contains a display that notifies the people about their medicine timings. Additionally, alerting with an alarm and light indications. To confirm whether the person has taken the medicines or not can be identified by putting a button at the opening end of the pillbox. When the person/patient is

Revised Manuscript Received on February 15, 2020

* Correspondence Author

Harshitha V*, Computer Science and Engineering, GITAM University, Bengaluru, India. Email: harshithahari1998@gmail.com

Sandeep K, Computer Science and Engineering, GITAM University, Bengaluru, India. Email: girijasandeep136@gmail.com

Swasthika Jain T J, Assistant Professor Computer Science and Engineering, GITAM University, Bengaluru, India. Email: swasthika.jain@gitam.edu

(C) The Authors. Published by Blue Eyes Intelligence Engineering and Sciences Publication (BEIESP). This is an open access article under the CC BY-NC-ND license (http://creativecommons.org/licenses/by-nc-nd/4.0/) feeling uneasy the different buttons fixed on the box he/she can press one of those fixed buttons to notify family members about the situation. [1][6] A Smart medicine box for the people who take medicine frequently and also remembering the prescription of the patient's medicine for a long time is very hard to remember by the patient and also the caretaker. Present time and notification time could be saved in RTC and EEPROM. [8] So, at the time of taking medicine, the system will be sending a notification and beep sound [4] and display the bright light in the pillbox from which the patient has to consume medicine. This system has an advantage of sensing whether the patient has consumed medicine or not and also senses when the patient tries to postpone the medication by opening and closing the box in to stop the notification and lighting. [2] An intelligent pillbox system is designed whether the patients have taken the correct dosage or not which has to be monitored by the caretaker. The caretaker will be notified by using the audio instructions when the medicines are taken or refilled. [10] Different functions that are present in this device are which pill has to be taken, the remainder of medicine timings to patient/caretaker and notifying using LED, buzzer, voice alarms, and the Android applications.[3]

A Pillbox with remind and consumption function that gives alert and box will be in open position at the time of taking medicines. [5] A device which has advanced futures like ability of sorting out the pills inside the box by itself and other advanced technologies are used here.[7] A Pillbox which reduces the family members responsibility and gets the feedback of the pills from the user and send the purchasing order to the medical shops. [9]

\section{SYSTEM DESIGN FOR PROPOSED METHODOLOGYK}

The below diagram depicts the components and their usage in the Interactive Pill Box System.

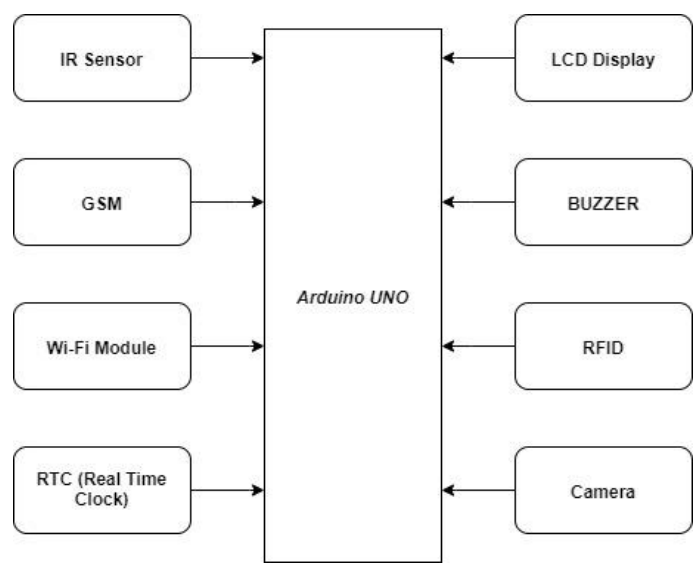

Fig 1: Block Diagram of an Interactive Pill Box System 


\section{A. WORKING}

An Interactive Pill Box System will not only reduce the work but helps to maintain the health of patients by taking the medication regularly at the prescribed time.

Sensors like IR (Infra-Red) sensor, camera, and RFID tags are used to count the number of pills inside a tray which are interfaced with the Arduino UNO micro-controller to make the system smarter. The RFID stickers fastened on each tablet sheet will be scanned by using the RFID, camera and IR sensor. The sensors will be giving the count of pills inside the box periodically for every 5 to 6 hours. The timings for the intake of medicines by the patient will be set for the device using RTC (Real Time Clock).

When the time for the medication to the patient has come the timer set for the device using RTC will be sending notification using buzzer and GSM to the patient and their family members. The notification will be sent to the mobile device and smartwatch which is connected to the device. By using RFID tags the pills which are taken can be identified whether the patient has taken correct medicine or not at a prescribed time. The data will be updated into the web browser using the Wi-Fi module. After completely taking the medicines over days/months the device will be fixing an appointment with the doctor automatically by sending a message using the GSM module and also convey the same to the medical shops to deliver the required medicines to patients address or to the hospitals where the patient stays. The same thing will be displayed on the 16x2 LCD.

\section{SYSTEM SPECIFICATIONS}

\section{A. ARDUINO UNO}

Arduino UNO is a microcontroller-based chip that controls all the units connected to it. The controller provides serial communication and it is designed in such a way that without requiring a physical reset button it allows reset by software running.

\section{B. IR SENSOR}

An infrared sensor is used to feel traits in its environment by way of emitting infrared radiation. It also senses the objects and their motion.

\section{GSM MODULE}

GSM module is used for communication purposes between mobile devices. It is developed at Bell Laboratories in the year 1970. It operates at different frequencies like $850 \mathrm{MHz}$, $900 \mathrm{MHz}, 1800 \mathrm{MHz}$ and $1900 \mathrm{MHz}$.

\section{Real-Time Clock}

It is an Integrated Chip (IC) that tracks the time. The chip is interfaced with a microprocessor circuit by an SPI serial bus.

\section{E. RFID}

Radio Frequency Identification uses electromagnetic radiations to identify objects. It consists of radio transponder which can transmit and receive.

\section{ALGORITHM USED}

In this, we will be using ID3 (Iterative Dichotomiser 3) to build a decision tree from examples to classify samples. Decision tree leaf nodes are class names and the non-leaf node is a decision node and this is a characteristic check with every branch being a likely cost of the attribute. The ID3 uses information gain which attributes goes into the decision nodes.

\section{A. ALGORITHM [11]}

1. Formulate Classification Attribute (in Table R).

2. Compute Classification Entropy.

3. Calculate Information Gain using classification attribute for each attribute $\mathrm{R}$.

4. The attribute which has the highest gain has to be selected as the next Node in the tree.

5. Node attribute has to be removed and create the reduced table RS.

6. Repeat steps 3 to 5 until all attributes are used. The classification values remain the same for all rows in the reduced table.

Entropy:

Information Gain:

$$
H(x)=-\sum_{i=1}^{N} P\left(x_{i}\right) \log _{b} P\left(x_{i}\right)
$$

For Set S, Attribute A

$$
I_{G}(S, A)=I_{E}(S)-\sum_{0}^{n}\left(P\left(\subset_{S}^{A_{K}}\right) * I_{E}\left(\subset_{S}^{A_{n}}\right)\right.
$$

\section{RESUlTS}

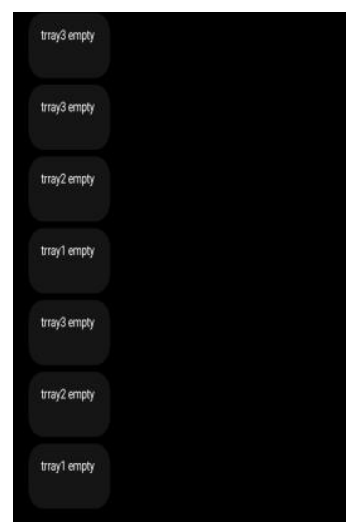

Fig 2: Alert Sent when the pills are empty in the tray

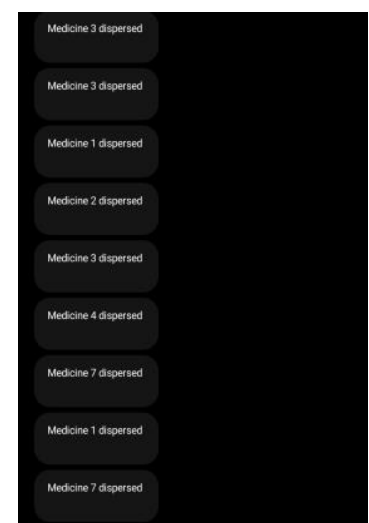

Fig 3: Alert Sent when the Medicine Dispensed from the tray

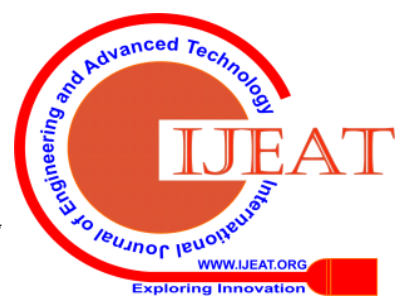


When the time for medication has come for the patients the device will be sending notification to the caretakers and also to the patients. [12]

\section{CONCLUSION}

This paper mainly focuses on alerting the users, care-takers about the medicine intake time of the patients. When the pill box becomes empty it sends a purchasing notification to the medical shop about the medicines that has to be purchased. The device also fixes an appointment with the doctor when the usage of medicines is completed. The main objective of these pill box is to overcome the problem of not remembering the medication timings of the patient is achieved through these IoT based smart pill box .

\section{REFERENCES}

1. Naga Swetha R, Mahendar, Roopsingh, Chinna, "Smart Pill Box Using IOT", Vol-5, Issue-4,2018.

2. Sanjay Bhati, Harshid Soni, Vijayrajsinh Zala, Parth Vyas, "Smart Medicine Reminder Box", Vol-3, Issue-10, April-2017.

3. Rushikesh Jadhav, Gajanan Bhople, Jyotsna Mahajan, Yogita Patil, "Intelligent Pillbox for Monitoring the Health using IOT Concepts", Vol-06, Issue-12, Dec-2019.

4. M Shailaja, K Lokeshwaran, S Sheik Faritha Begum, "Smart Medication Pill Box for Blind People with Pulse Sensor", Vol-8, Issue-1S2, May-2019.

5. L Sangavi, M Vitharcchana, B Sivachalapathy, M Shanmugham, "An Intelligent Pill Box with Reminder Using IOT”, Vol-3, Issue-1, 2018.

6. Jayesh Patil, Sameer Khairmode, Rishikesh Lokhande, Omkar Shinde, "The Autonomous Pill Dispenser with Alarm and Mobile Notifications", Vol-7, Issue-4, April-2019.

7. Mohamed Abd-ELfattah, Diaa Salama Abdul Minaam, "Smart drugs: Improving healthcare using Smart Pill Box for Medicine Reminder and Monitoring System", Future Computing and Informatics Journal 3 (2018) 443-456

8. Nausheen Fatima, Praveen Hipparge, "IOT Driven Smart Pill Box to Remind of Consumption", Vol-06, Issue-06, June-2019.

9. Shashank Shinde, Tejas Kadaskar, Pushpak Patil, Rohit Barathe, "A Smart Pill Box with Remind and Consumption Using IOT", Vol-04, Issue-12, Dec-2017.

10. Hsiu-Ling Tsai, Chun Hsiang Tseng, Long-Cian Wang, Fuh-Shyang Juang, "Bidirectional Smart Pill Box Monitored

11. Through Internet and Receiving Reminding Message from Remote Relatives", ICCE-Taiwan, 2017.

12. Viral Doshi, Nirav Mehta, Shrishti Dey, Rajesh Prasad, "An IoT Based Smart Medicine Box", Vol-5, Issue-1, 2019.

13. Nidhi Solanki, DR.P.H. Zope, "Smart PillBox Health Care System", Vol-05, Issue-07, July 2018.

\section{AUTHORS PROFILE}

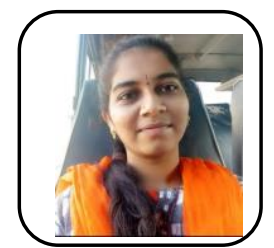

Harshitha V, is a student pursuing her B. Tech Final year of Computer Science and Engineering in GITAM (Deemed to be University), Bengaluru, Karnataka, India. Her Project Smart Pillbox using IoT has been awarded first prize in the Engineers day celebration conducted by GUSAC in GITAM University Bengaluru on September 15, 2019. Currently she is doing her project on IoT based smart robot for disaster management applications. She has been certified as a Microsoft Technical Associate (MTA) by Microsoft on introduction of Python with Machine Learning. She has done an Autonomous Robot design in microcontroller based AutoBotix Platform, Robot Programming using ASM and C language.

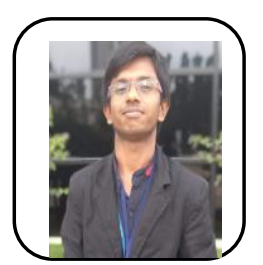

Sandeep K, is a student pursuing his B. Tech Final year of Computer Science and Engineering in GITAM (Deemed to be University), Bengaluru, Karnataka, India. Sandeep has done his research in different fields like neural networks, image processing and has published paper on cyber security using neural networks in international conference conducted by Garden City University, Bengaluru. His project image processing using MATLAB for blind people has been selected as one in top 20 for Student Innovation Award- 2018, Karnataka which is conducted by ICT academy. His project smart pillbox using IoT has been awarded first prize in engineers day celebration conducted by GUSAC in GITAM University Bengaluru on September 15, 2019.

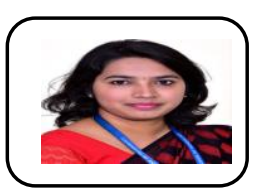

Swasthika Jain T J, currently working as Assistant Professor, Computer science and Engineering. GITAM School of Technology, Bengaluru Campus. Overall, she is having 6 years of teaching experience. She did her BE in Information Science and Engineering from Bahubali College of Engineering and her M. Tech in Computer Science and Engineering from Rajiv Gandhi Institute of Technology. She is pursuing her Ph.D. in computer Science and Engineering. Her area of research is Big data analytics. She published papers in different areas like data analytics, wireless sensor network, Internet of Things and she is certified trainee associate by Dell EMC for data analytics with R. 